\title{
MORE ON STRONG SIZE PROPERTIES
}

\author{
Sergio Macías and CÉsar Piceno \\ Universidad Nacional Autónoma de México, México
}

\begin{abstract}
We continue our study of strong size maps. We show that strong size levels for the $n$-fold hyperspace of a continuum contain $(n-1)$-cells. We give two constructions of strong size maps. We introduce reversible strong size properties. We prove that each of the following properties: being a continuum chainable continuum, being a locally connected continuum, and being a continuum with the property of Kelley, is a reversible strong size property. Following Professors Goodykoontz and Nadler, we define admissible strong size maps and show that the levels of admissible strong size maps for the $n$-fold hyperspace of a locally connected continuum are homeomorphic to the Hilbert cube. Professor Benjamín Espinoza defined Whitney preserving maps for the hyperspace of subcontinua of a continuum. We define strong size preserving maps and show that this class of maps coincides with the class of homeomorphisms.
\end{abstract}

\section{INTRODUCTION}

We continue our study of strong size maps ([17]). Professor H. Hosokawa defines strong size maps for the $n$-fold hyperspace of a continuum $X$ in [11] as a natural generalization of a Whitney map for the hyperspace of subcontinua of $X$. We characterize strong size levels for the $n$-fold hyperspace of a continuum as antichains that are intersected by order arcs (Theorem 3.1) and prove that such levels contain $(n-1)$-cells (Corollary 3.3$)$. We present two constructions of strong size maps (Examples 4.1 and 4.2). We introduce reversible strong size properties and show that each of the following properties is a reversible strong size property: being a continuum chainable continuum

2010 Mathematics Subject Classification. 54B20.

Key words and phrases. Absolute retract, acyclic continuum, admissible strong size map, continuum, continuum chainable continuum, Hilbert cube, $n$-fold hyperspace, $n$-fold symmetric product, retract, retraction, reversible strong size property, strong size level, strong size map, strong size properties. 
(Theorem 5.6), being a locally connected continuum (Theorem 5.7) and being a continuum with the property of Kelley (Theorem 5.8). Following Professors Goodykoontz and Nadler ([9]), we define admissible strong size maps and show that the levels of admissible strong size maps for the $n$-fold hyperspace of a locally connected continuum are homeomorphic to the Hilbert cube (Theorem 6.22). Professor Benjamín Espinoza defines Whitney preserving maps for the hyperspace of subcontinua of a continuum in [8]. We define strong size preserving maps and show that this class of maps coincides with the class of homeomorphisms (Theorem 7.4).

In some of our results we include a citation, that means that our theorem is similar to the one cited, in most cases, we include the proof for the convenience of the reader.

\section{Definitions AND NOTATION}

If $(Z, d)$ is a metric space, then given a subset $A$ of $Z$ and $\varepsilon>0$, the open ball about $A$ of radius $\varepsilon$ is denoted by $\mathcal{V}_{\varepsilon}^{d}(A)$, the interior of $A$ is denoted by $\operatorname{Int}_{Z}(A)$, the boundary of $A$ is denoted by $B d_{Z}(A)$, and the closure of $A$ is denoted by $C l_{Z}(A)$. The identity map on $Z$ is denoted by $1_{Z}$.

By a map we mean a continuous function. Let $Z$ be a metric space and $A$ a nonempty subset of $Z$. Then a map $r: Z \rightarrow A$ is a retraction provided that $r(a)=a$ for all $a \in A$. In this case $A$ is a retract of $Z$.

Let $Z$ be a metric space. By a deformation we mean a map $H: Z \times$ $[0,1] \rightarrow Z$ such that $H((z, 0))=z$ for each $z \in Z$. Let $A=\{H((z, 1)) \mid z \in$ $Z\}$. If the map $r: Z \rightarrow A$ given by $r(z)=H((z, 1))$ is a retraction from $Z$ onto $A$, then $H$ is a deformation retraction from $Z$ onto $A$. If $H$ is a deformation retraction from $Z$ onto $A$ and for each $a \in A$ and each $t \in[0,1]$, $H((a, t))=a$, then $H$ is a strong deformation retraction from $Z$ onto $A$. The set $A$ is called a deformation retract of $Z$ (strong deformation retract of $Z$, respectively). A metric space $Z$ is an absolute retract provided that for each metric space $Y$ and each embedding $e: Z \rightarrow Y$ of $Z$ into $Y$ such that $e(Z)$ is a closed subset of $Y, e(Z)$ is a retract of $Y$.

A continuum is a nonempty compact connected metric space.

Given a continuum $X$ and two points $x_{1}$ and $x_{2}$ of $X$, a finite collection $\left\{L_{1}, \ldots, L_{m}\right\}$ of subsets of $X$ is called a chain from $x_{1}$ to $x_{2}$ provided that $x_{1} \in L_{1}, x_{2} \in L_{m}$ and $L_{i} \cap L_{j} \neq \emptyset$ if and only if $|i-j| \leq 1$. A chain is called a continuum chain if each of its elements is a continuum. A continuum chain is an $\varepsilon$-continuum chain if the diameter of each of its elements is less than $\varepsilon$. The continuum $X$ is said to be continuum chainable continuum provided that for each pair of points $x_{1}$ and $x_{2}$ of $X$ and each $\varepsilon>0$, there exists an $\varepsilon$-continuum chain from $x_{1}$ to $x_{2}$. 
An arc is any space homeomorphic to [0,1], the end points of an arc are the images of 0 and 1 under a homeomorphism. A continuum $X$ is arcwise connected if each pair of its points can be joined by an $\operatorname{arc}$ in $X$.

REMARK 2.1. Observe that every arcwise connected continuum is a continuum chainable continuum.

If $X$ is a continuum and $n$ is a positive integer, then $\check{H}^{n}(X)$ denotes the reduced $n$th Cech cohomology group of $X$ with integer coefficients. A continuum $X$ is said to be acyclic if $\check{H}^{1}(X)$ is trivial.

Given a compactum $X$, we consider the following hyperspaces of $X$ :

$$
2^{X}=\{A \subset X \mid A \text { is nonempty and closed }\}
$$

and

$$
\mathcal{C}_{n}(X)=\left\{A \in 2^{X} \mid A \text { has at most } n \text { components }\right\},
$$

where $n$ is a positive integer. $\mathcal{C}_{n}(X)$ is called the $n$-fold hyperspace of $X$. These spaces are topologized with the Hausdorff metric defined as follows:

$$
\mathcal{H}(A, B)=\inf \left\{\varepsilon>0 \mid A \subset \mathcal{V}_{\varepsilon}^{d}(B) \text { and } B \subset \mathcal{V}_{\varepsilon}^{d}(A)\right\},
$$

$\mathcal{H}$ always denotes the Hausdorff metric on $2^{X}$. When $n=1$, we write $\mathcal{C}(X)$ instead of $\mathcal{C}_{1}(X)$. Given an element $B \in \mathcal{C}_{n}(X)$, the mesh of $B$, denoted by $\operatorname{mesh}(B)$, is

$$
\operatorname{mesh}(B)=\max \{\operatorname{diam}(K) \mid K \text { is a component of } B\} .
$$

The symbol $\mathcal{F}_{n}(X)$ denotes the $n$-fold symmetric product of $X$; that is:

$$
\mathcal{F}_{n}(X)=\left\{A \in \mathcal{C}_{n}(X) \mid A \text { has at most } n \text { points }\right\} .
$$

Note that, by definition, $\mathcal{F}_{n}(X) \subset \mathcal{C}_{n}(X)$. It is known, that if $X$ is a continuum, then $2^{X}$ and $\mathcal{C}_{n}(X)$ are arcwise connected continua (for $2^{X}$ and $\mathcal{C}(X)$ see $[19,(1.13)]$; for $\mathcal{C}_{n}(X)$ and $n \geq 2$, see $\left.[16,1.8 .12]\right)$. Also, $\mathcal{F}_{n}(X)$ is a continuum for all positive integers $n([3$, p. 877]). Let $X$ be a continuum and let $n$ be a positive integer. An order arc in $\mathcal{C}_{n}(X)$ is an arc $\alpha:[0,1] \rightarrow \mathcal{C}_{n}(X)$ such that if $0 \leq s<t \leq 1$, then $\alpha(s) \subset \alpha(t)$ and $\alpha(s) \neq \alpha(t)$.

Let $X$ a continuum and let $n$ a positive integer. If $B \in \mathcal{C}_{n}(X)$, define:

$$
\mathcal{C}_{n}(B, X)=\left\{A \in \mathcal{C}_{n}(X) \mid B \subset A\right\} .
$$

A map $\mu: \mathcal{C}_{n}(X) \rightarrow[0,1]$ is a size map if $\mu(\{x\})=0$ for all $x \in X$; if $A, B \in \mathcal{C}_{n}(X)$ and $A \subset B$, then $\mu(A) \leq \mu(B)$, and $\mu(X)=1$.

A map $\mu: \mathcal{C}_{n}(X) \rightarrow[0,1]$ is said to be a strong size map provided that $\mu(A)=0$ for each $A \in \mathcal{F}_{n}(X)$; if $A, B \in \mathcal{C}_{n}(X), A \subset B, A \neq B$ and $B \notin \mathcal{F}_{n}(X)$, then $\mu(A)<\mu(B)$, and $\mu(X)=1$.

By [11, Theorem 2.2], strong size maps exist for each continuum $X$ and each positive integer $n$. Note that for $n=1$ a strong size map is just a Whitney map (see [19, (0.50)] for definition). 
Let $X$ be a continuum, let $n$ be a positive integer and let $\mu: \mathcal{C}_{n}(X) \rightarrow[0,1]$ be a strong size map. A strong size level of $\mu$ in $\mathcal{C}_{n}(X)$ is a set of the form $\mu^{-1}(t)$ for some $t \in[0,1]$.

A topological property $P$ is called strong size property if whenever the continuum $X$ has property $P$, then for every positive integer $n$ and each strong size map $\mu$ for $\mathcal{C}_{n}(X)$, we have that every strong size level of $\mu$ in $\mathcal{C}_{n}(X)$ has property $P$.

Let $X$ be a continuum and let $\mathcal{S}$ be a subset of $\mathcal{C}_{n}(X) \backslash \mathcal{F}_{n}(X)$. Then $\mathcal{S}$ is said to be an antichain if given two elements $S$ and $S^{\prime}$ of $\mathcal{S}$ such that $S \subset S^{\prime}$, then $S=S^{\prime}$.

\section{Properties of STRONG Size LEVELS}

TheOREM 3.1 ([14, 23.8]). Let $X$ be a nondegenerate continuum. Then a nonempty closed subset $\mathcal{S}$ of $\mathcal{C}_{n}(X)$ is a strong size level if and only if either $\mathcal{S}=\mathcal{F}_{n}(X)$ or $\mathcal{S} \cap \mathcal{F}_{n}(X)=\emptyset, \mathcal{S}$ is an antichain and every order arc from an element of $\mathcal{F}_{n}(X)$ to $X$ intersects $\mathcal{S}$.

Proof. If $\mathcal{S}$ is a strong size level, it is clear that either $\mathcal{S}=\mathcal{F}_{n}(X)$ or $\mathcal{S} \cap \mathcal{F}_{n}(X)=\emptyset, \mathcal{S}$ is an antichain and every order arc from an element of $\mathcal{F}_{n}(X)$ to $X$ intersects $\mathcal{S}$.

Suppose that $\mathcal{S} \cap \mathcal{F}_{n}(X)=\emptyset, \mathcal{S}$ is an antichain and every order arc from an element of $\mathcal{F}_{n}(X)$ to $X$ intersects $\mathcal{S}$.

Define $\mu^{\prime}: \mathcal{S} \rightarrow[0,1]$, by $\mu^{\prime}(S)=\frac{1}{2}$ for each $S \in \mathcal{S}$. Observe that $\mu^{\prime}$ is a strong size map for $\mathcal{S}$. Hence, by [17, Theorem 5.4], there exists a strong size $\operatorname{map} \mu: \mathcal{C}_{n}(X) \rightarrow[0,1]$ such that $\left.\mu\right|_{\mathcal{S}}=\mu^{\prime}$. By construction, $\mathcal{S} \subset \mu^{-1}\left(\frac{1}{2}\right)$. To show that $\mu^{-1}\left(\frac{1}{2}\right) \subset \mathcal{S}$, let $A \in \mu^{-1}\left(\frac{1}{2}\right)$ and let $\alpha_{A}:[0,1] \rightarrow \mathcal{C}_{n}(X)$ be an order arc from some point of $\mathcal{F}_{n}(X)$ to $X$ passing through $A$, by hypothesis, $\alpha_{A}([0,1]) \cap \mathcal{S} \neq \emptyset$, let $A^{\prime} \in \alpha_{A}([0,1]) \cap \mathcal{S}$. Since $\mu$ is a strong size map, $A^{\prime} \in \mu^{-1}\left(\frac{1}{2}\right)$, and either $A^{\prime} \subset A$ or $A \subset A^{\prime}$, we have that $A=A^{\prime}$. Therefore, $\mathcal{S}=\mu^{-1}\left(\frac{1}{2}\right)$.

If $\mathcal{S}=\mathcal{F}_{n}(X)$, then $\mathcal{S}=\mu^{-1}(0)$ for each strong size map $\mu$.

TheOrem 3.2. Let $X$ be a continuum, let $n \geq 2$, let $\mu: \mathcal{C}_{n}(X) \rightarrow[0,1]$ be a strong size map and let $t \in(0,1)$. If $A \in\left(\mathcal{C}_{n}(X) \backslash \mathcal{C}_{n-1}(X)\right) \cap \mu^{-1}(t)$, then there exist an $(n-1)$-cell $\mathcal{A}$ contained in $\mu^{-1}(t)$ such that $A \in \mathcal{A}$.

Proof. Let $A \in\left(\mathcal{C}_{n}(X) \backslash \mathcal{C}_{n-1}\right) \cap \mu^{-1}(t)$. Let $A_{1}, \ldots, A_{n}$ be the components of $A$. For each $j \in\{1, \ldots, n\}$, let $a_{j}$ be a point of $A_{j}$, and let $\alpha_{j}:[0,1] \rightarrow \mathcal{C}(X)$ be an order arc such that $\alpha_{j}(0)=\left\{a_{j}\right\}$, $\alpha_{j}(1)=X$ and $\alpha_{j}\left(\frac{1}{2}\right)=A_{j}$. Define the function $\xi:[0,1]^{n} \rightarrow \mathcal{C}_{n}(X)$ by $\xi\left(\left(r_{1}, \ldots, r_{n}\right)\right)=\bigcup_{j=1}^{n} \alpha_{j}\left(r_{j}\right)$. Then $\xi$ is well defined and continuous. Since $A \in \mathcal{C}_{n}(X) \backslash \mathcal{C}_{n-1}(X)$ and $\xi$ is continuous, there exists an $\varepsilon>0$ such that 
$\xi\left(\prod_{j=1}^{n}\left[\frac{1}{2}-\varepsilon, \frac{1}{2}+\varepsilon\right]_{j}\right) \subset \mathcal{C}_{n}(X) \backslash \mathcal{C}_{n-1}(X)$, where $\left[\frac{1}{2}-\varepsilon, \frac{1}{2}+\varepsilon\right]_{j}$ is a copy of $\left[\frac{1}{2}-\varepsilon, \frac{1}{2}+\varepsilon\right]$.

Note that $A=\xi\left(\left(\frac{1}{2}, \ldots, \frac{1}{2}\right)\right)$ and $\mu\left(\xi\left(\left(\frac{1}{2}, \ldots, \frac{1}{2}+\varepsilon\right)\right)\right)>t$. By the continuity of $\alpha$ and $\mu$, there exists a $\delta>0$ such that $\delta<\varepsilon$ and

$$
\mu\left(\xi\left(\left(s_{1}, \ldots, s_{n-1}, \frac{1}{2}+\varepsilon\right)\right)\right)>t
$$

for all $\left(s_{1}, \ldots, s_{n-1}\right) \in \prod_{j=1}^{n-1}\left[\frac{1}{2}-\delta, \frac{1}{2}+\delta\right]_{j}$.

Define $\theta: \prod_{j=1}^{n-1}\left[\frac{1}{2}-\delta, \frac{1}{2}\right]_{j} \rightarrow\left[0, \frac{1}{2}+\varepsilon\right]$ as follows: For each point $\left(s_{1}, \ldots, s_{n-1}\right)$ of $\prod_{j=1}^{n-1}\left[\frac{1}{2}-\delta, \frac{1}{2}\right]_{j}$, let $\theta\left(\left(s_{1}, \ldots, s_{n-1}\right)\right)$ be the unique element of $\left[0, \frac{1}{2}+\varepsilon\right]$ such that $\mu\left(\xi\left(\left(s_{1}, \ldots, s_{n-1}\right), \theta\left(\left(s_{1}, \ldots, s_{n-1}\right)\right)\right)\right)=t$.

To prove that $\theta$ is a continuous function, let $\left\{\left(s_{1}^{k}, \ldots, s_{n-1}^{k}\right)\right\}_{k=1}^{\infty}$ be a sequence of elements of $\prod_{j=1}^{n-1}\left[\frac{1}{2}-\delta, \frac{1}{2}\right]_{j}$ converging to $\left(s_{1}, \ldots, s_{n-1}\right) \in$ $\prod_{j=1}^{n-1}\left[\frac{1}{2}-\delta, \frac{1}{2}\right]_{j}$. Consider the sequence $\left\{\theta\left(\left(s_{1}^{k}, \ldots, s_{n-1}^{k}\right)\right)\right\}_{k=1}^{\infty}$ of elements of $\left[0, \frac{1}{2}+\varepsilon\right]$. Since this set is compact, $\left\{\theta\left(\left(s_{1}^{k}, \ldots, s_{n-1}^{k}\right)\right)\right\}_{k=1}^{\infty}$ has a convergent subsequence $\left\{\theta\left(\left(s_{1}^{k_{\ell}}, \ldots, s_{n-1}^{k_{\ell}}\right)\right)\right\}_{\ell=1}^{\infty}$ converging to $s \in\left[0, \frac{1}{2}+\varepsilon\right]$. Then, since $\mu$ and $\xi$ are continuous, we have:

$$
\begin{aligned}
\lim _{\ell \rightarrow \infty} & \mu\left(\xi\left(\left(s_{1}^{k_{\ell}}, \ldots, s_{n-1}^{k_{\ell}}\right), \theta\left(\left(s_{1}^{k_{\ell}}, \ldots, s_{n-1}^{k_{\ell}}\right)\right)\right)\right) \\
& =\mu\left(\xi\left(\lim _{\ell \rightarrow \infty}\left(s_{1}^{k_{\ell}}, \ldots, s_{n-1}^{k_{\ell}}\right), \lim _{\ell \rightarrow \infty} \theta\left(\left(s_{1}^{k_{\ell}}, \ldots, s_{n-1}^{k_{\ell}}\right)\right)\right)\right) \\
& =\mu\left(\xi\left(\left(s_{1}, \ldots, s_{n-1}, s\right)\right)\right) .
\end{aligned}
$$

Since for each positive integer $\ell, \mu\left(\xi\left(\left(s_{1}^{k_{\ell}}, \ldots, s_{n-1}^{k_{\ell}}\right), \theta\left(\left(s_{1}^{k_{\ell}}, \ldots, s_{n-1}^{k_{\ell}}\right)\right)\right)\right)=$ $t$, we obtain that $\mu\left(\xi\left(\left(s_{1}, \ldots, s_{n-1}, s\right)\right)\right)=t$ and $\theta\left(\left(s_{1}, \ldots, s_{n-1}\right)\right)=s$. Hence, $\theta$ is continuous.

Let $\chi: \prod_{j=1}^{n-1}\left[\frac{1}{2}-\delta, \frac{1}{2}\right]_{j} \rightarrow \mu^{-1}(t)$ be given by

$$
\chi\left(\left(s_{1}, \ldots, s_{n-1}\right)\right)=\xi\left(\left(s_{1}, \ldots, s_{n-1}\right), \theta\left(\left(s_{1}, \ldots, s_{n-1}\right)\right)\right) .
$$

Then $\chi$ is an embedding of the $(n-1)$-cell $\prod_{j=1}^{n-1}\left[\frac{1}{2}-\delta, \frac{1}{2}\right]_{j}$ into $\mu^{-1}(t)$.

Corollary 3.3. Let $X$ be a continuum, let $n \geq 2$, let $\mu: \mathcal{C}_{n}(X) \rightarrow[0,1]$ be a strong size map and let $t \in(0,1)$. Then $\mu^{-1}(t)$ contains an $(n-1)$-cell.

Proof. Since $\mathcal{C}_{n-1}(X)$ has empty interior in $\mathcal{C}_{n}(X)([16,6.1 .7])$, we have that $\mu^{-1}(t) \cap\left(\mathcal{C}_{n}(X) \backslash \mathcal{C}_{n-1}(X)\right) \neq \emptyset$. Now, the corollary follows from Theorem 3.2 .

COROLlaRY 3.4. The property of being a hereditarily indecomposable continuum is not a strong size property. 


\section{Constructions of Strong size maps}

It is shown in [11] the existence of the strong size maps, but the author does not give a construction of them. In this section, we present the construction of two strong size map for every positive integer $n$. Example 4.2 is used in the proof of Theorem 6.14. Also, we give two algebraic properties of the strong size maps.

The next example is taken from [22, pp. 275-276].

EXAMPLE 4.1. Let $X$ be a continuum with a bounded metric $d$ bounded by 1 , let $n$ be a positive integer and let $A \in \mathcal{C}_{n}(X)$. For each integer $m \geq 2$ and $K \in \mathcal{F}_{m}(A)$, where $K=\left\{k_{1}, \ldots, k_{m}\right\}$, it is possible that $k_{j}=k_{l}$ for $j \neq l$. Define $\omega_{m}(K)=\min \left\{d\left(k_{j}, k_{l}\right) \mid j \neq l\right\}$. Define $\mu_{m}(A)=\sup \left\{\omega_{m}(K) \mid K \in \mathcal{F}_{m}(A)\right\}$. Then the function $\mu: \mathcal{C}_{n}(X) \rightarrow[0,1]$ given by $\mu(A)=\sum_{j=n+1}^{\infty} \frac{1}{2^{j}} \mu_{j}(A)$ is a strong size map. To see this, note that $\mu$ is continuous. Observe that if $A \in \mathcal{F}_{n}(X)$, then, clearly, $\mu_{m}(A)=0$ for all $m>n$. This implies that $\mu(A)=0$. Now, if $A \in \mathcal{C}_{n}(X) \backslash \mathcal{F}_{n}(X)$, then $A$ has more than $n+1$ elements. Hence, $\mu_{n+1}(A)>0$, and $\mu(A)>0$. Now, suppose $A$ and $B$ are two elements of $\mathcal{C}_{n}(X)$ such that $A \subsetneq B$ and $B \in \mathcal{C}_{n}(X) \backslash \mathcal{F}_{n}(X)$. The proof of the fact that $\mu(A)<\mu(B)$ is similar to the argument given in [22, pp. 275-276].

The next example is taken from [12].

EXAMPLE 4.2 . Let $X$ be a continuum and let $n$ be a positive integer. For each $A \in \mathcal{C}_{n}(X)$, let

$\mu_{m}(A)=\inf \left\{\varepsilon>0 \mid\right.$ there exist $p_{1}, \ldots, p_{m} \in X$ such that $\left.A \subset \bigcup_{j=1}^{m} \mathcal{V}_{\varepsilon}\left(p_{j}\right)\right\}$.

Then $\mu: \mathcal{C}_{n}(X) \rightarrow[0,1]$ given by $\mu(A)=\sum_{m=n}^{\infty} \frac{1}{2^{m}} \mu_{m}(A)$ is a strong size map. To show this, note $\mu$ is continuous. Observe that if $A \in \mathcal{F}_{n}(X)$, then, clearly, $\mu_{m}(A)=0$ for all $m>n$. This implies that $\mu(A)=0$. If $A \in \mathcal{C}_{n}(X) \backslash \mathcal{F}_{n}(X)$, then $A$ has more than $n$ elements. Thus, $\mu_{n}(A)>0$, and $\mu(A)>0$. Now, suppose $A$ and $B$ are two elements of $\mathcal{C}_{n}(X)$ such that $A \subsetneq B$ and $B \in \mathcal{C}_{n}(X) \backslash \mathcal{F}_{n}(X)$. Without loss of generality, we assume that $A \in \mathcal{C}_{n}(X) \backslash \mathcal{F}_{n}(X)$ also. Let $b \in B \backslash A$ and let $\varepsilon>0$ be such that $d(b, A)>2 \varepsilon$ and we assume $\varepsilon$ is small enough that we need more than $n$ points of $X$ to cover $A$ with open balls of radius $\varepsilon$. Let

$$
\ell=\min \left\{m \geq n \mid \text { there exist } x_{1}, \ldots, x_{m} \in X \text { such that } A \subset \bigcup_{j=1}^{m} \mathcal{V}_{\varepsilon}\left(x_{j}\right)\right\} \text {. }
$$

Since $A$ is compact, there exists $\delta>0$ such that $\delta<\varepsilon$ and $A \subset \bigcup_{j=1}^{m} \mathcal{V}_{\delta}\left(x_{j}\right)$. This implies that $\mu_{\ell}(A)<\varepsilon$. We claim that $\mu_{\ell}(B) \geq \varepsilon$. Suppose this is not 
true and assume that $\mu_{\ell}(B)<\varepsilon$. Then there exist $b_{1}, \ldots, b_{\ell} \in X$ such that $B \subset \bigcup_{j=1}^{\ell} \mathcal{V}_{\varepsilon}\left(b_{j}\right)$. Note that $b \in \bigcup_{j=1}^{\ell} \mathcal{V}_{\varepsilon}\left(b_{j}\right)$. Without loss of generality, we assume that $b \in \mathcal{V}_{\varepsilon}\left(b_{\ell}\right)$. Then $A \subset \bigcup_{j=1}^{\ell-1} \mathcal{V}_{\varepsilon}\left(b_{j}\right)$, a contradiction to the election of $\ell$. Thus, $\mu_{\ell}(B) \geq \varepsilon$. Therefore, $\mu_{\ell}(A)<\mu_{\ell}(B)$.

Lemma 4.3. Let $X$ be a continuum, let $n$ be a positive integer, and let $\mu: \mathcal{C}_{n}(X) \rightarrow[0,1]$ be a strong size map. If $\omega: \mathcal{C}_{n}(X) \rightarrow[0,1]$ is a size map such that $\omega(A)>0$ for all $A \in \mathcal{C}_{n}(X) \backslash \mathcal{F}_{n}(X)$, then $\mu \cdot \omega: \mathcal{C}_{n}(X) \rightarrow[0,1]$ is a strong size map.

Proof. Clearly, $\mu \cdot \omega$ is well defined and is continuous. To see that is a strong size map, let $A \in \mathcal{F}_{n}(X)$. Then $(\mu \cdot \omega)(A)=\mu(A) \cdot \omega(A)=0 \cdot \omega(A)=0$. Now, suppose $A$ and $B$ are two elements of $\mathcal{C}_{n}(X)$ such that $A \subsetneq B$ and $B \in \mathcal{C}_{n}(X) \backslash \mathcal{F}_{n}(X)$. Then $(\mu \cdot \omega)(A)=\mu(A) \cdot \omega(A) \leq \mu(A) \cdot \omega(B)<$ $\mu(B) \cdot \omega(B)=(\mu \cdot \omega)(B)$ (the first inequality holds since $\omega(B)>0$ ). Therefore, $\mu \cdot \omega$ is a strong size map.

\section{ReVERsible Strong SIZE PROPERTIEs}

A topological property $P$ is an $n$-reversible property provided that whenever $X$ is a continuum such that $\mu^{-1}(t)$ has property $P$ for some positive integer $n$ fixed, for all strong size maps $\mu: \mathcal{C}_{n}(X) \rightarrow[0,1]$ and all $0 \leq t<1$, then $X$ has property $P$.

REMARK 5.1. Note that a topological property $P$ is 1-reversible if and only if $P$ is a Whitney-reversible property (see [19, (14.45)] for the definition).

Remark 5.2. Since for each continuum $X$ and every $n \geq 3$, the strong size levels of each strong size map are acyclic [17, Theorem 4.16], we have that being acyclic is not an $n$-reversible property for any $n \geq 3$.

QUESTION 5.1. Is the property of being an acyclic continuum a 2reversible property?

Question 5.2. Let $n$ and $m$ be positive integers such that $m<n$. Does there exist an $n$-reversible property that is not an $m$-reversible property?

A topological property $P$ is a powerful strong size-reversible property provided that if $X$ is a continuum such that there exist a positive integer $n$ and a strong size map $\mu: \mathcal{C}_{n}(X) \rightarrow[0,1]$ such that $\mu^{-1}(t)$ has property $P$ for all $0 \leq t<1$, then $X$ has property $P$.

Lemma 5.3. Let $X$ be a continuum, let $n$ be a positive integer and let $\mu: \mathcal{C}_{n}(X) \rightarrow[0,1]$ be a strong size map. Then for each $\varepsilon>0$, there exists $t>0$ such that $\operatorname{mesh}(A)<\varepsilon$ for all $A \in \mu^{-1}(t)$.

Proof. Suppose the lemma is not true. Then there exists $\varepsilon>0$ such that for each positive integer $m$, there exists $A_{m} \in \mu^{-1}\left(\frac{1}{m}\right) \operatorname{such}$ that $\operatorname{mesh}\left(A_{m}\right)>$ 
$\varepsilon$. For every positive integer $m$, let $A_{m}^{\prime}$ be a component of $A_{m}$ such that $\operatorname{diam}\left(A_{m}^{\prime}\right)>\varepsilon$. Since $\mathcal{C}(X)$ is compact $([16,1.8 .5])$, without loss of generality, we assume that the sequence $\left\{A_{m}^{\prime}\right\}_{m=1}^{\infty}$ converges to an element $A^{\prime} \in \mathcal{C}(X)$. Since $\operatorname{diam}\left(A_{m}^{\prime}\right)>\varepsilon$ for all $m, \operatorname{diam}\left(A^{\prime}\right) \geq \varepsilon$. Since $A_{m}^{\prime} \subset A_{m}$ and $\mu\left(A_{m}\right)<$ $\frac{1}{m}$ for each $m$, we have that $\mu\left(A^{\prime}\right)=0$. Thus, $A^{\prime} \in \mathcal{F}_{1}(X)$ and $\operatorname{diam}\left(A^{\prime}\right)=0$, a contradiction. Therefore, the lemma is true.

LEMma 5.4. Let $X$ be a continuum, let $n$ be a positive integer, and let $\varepsilon>0$. If $A \in \mathcal{C}_{n}(X)$ is such that $\operatorname{mesh}(A)<\varepsilon$, and $\mathcal{A}$ is a subcontinuum of $\mathcal{C}_{n}(X)$ such that $A \in \mathcal{A}$ and $\operatorname{diam}(\mathcal{A})<\varepsilon$, then mesh $(\cup \mathcal{A}) \leq 3 n \varepsilon$.

Proof. Let $A \in \mathcal{C}_{n}(X)$ and let $\mathcal{A}$ be a subcontinuum of $\mathcal{C}_{n}(X)$ such that $A \in \mathcal{A}$ and $\operatorname{diam}(\mathcal{A})<\varepsilon$. Since $A \in \mathcal{A}$ and $\operatorname{diam}(\mathcal{A})<\varepsilon, \mathcal{H}^{2}(\{A\}, \mathcal{A})<\varepsilon$, where $\mathcal{H}^{2}$ is the Hausdorff metric on $\mathcal{C}\left(\mathcal{C}_{n}(X)\right)$, we have that $\mathcal{H}(A, \cup \mathcal{A})<\varepsilon$ $([19,(1.48)])$. Let $A_{1}, \ldots, A_{m}$ be the components of $A$. Since $\mathcal{H}(A, \bigcup \mathcal{A})<\varepsilon$, we have that $\bigcup \mathcal{A} \subset \bigcup_{j=1}^{m} \mathcal{V}_{\varepsilon}\left(A_{j}\right)$. Hence, if $D$ is a component of $\bigcup \mathcal{A}$, then $\operatorname{diam}(D) \leq \sum_{j=1}^{m} \operatorname{diam}\left(\mathcal{V}_{\varepsilon}\left(A_{j}\right)\right) \leq m(3 \varepsilon) \leq 3 n \varepsilon$.

The following lemma is a direct consequence of [10, Lemma 3.1].

Lemma 5.5. Let $X$ be a continuum and let $n$ be a positive integer. If $\mathcal{A}$ and $\mathcal{B}$ are two subcontinua of $\mathcal{C}_{n}(X)$ such that $\mathcal{A} \cap \mathcal{B} \neq \emptyset$. Then each component of $\bigcup \mathcal{A}$ intersects $\bigcup \mathcal{B}$ and each component of $\cup \mathcal{B}$ intersects $\cup \mathcal{A}$.

THEOREM 5.6. The property of being a continuum chainable continuum is a powerful strong size-reversible property.

Proof. Let $X$ be a continuum, let $n$ be a positive integer and let $\mu: \mathcal{C}_{n}(X) \rightarrow[0,1]$ be a strong size map such that $\mu^{-1}(r)$ is a continuum chainable continuum for all $r \in[0,1)$. Let $\varepsilon>0$. By Lemma 5.3, there exist $t>0$ such that $\operatorname{mesh}(A)<\frac{\varepsilon}{3 n}$ for each $A \in \mu^{-1}(t)$. Let $p$ and $q$ be two elements of $X$ and $P$ and $Q$ be two elements of $\mathcal{C}(X) \cap \mu^{-1}(t)$ such that $p \in P$ and $q \in Q$. Since $\mu^{-1}(t)$ is a continuum chainable continuum, there exist an $\frac{\varepsilon}{3 n}$-chain $\left\{\mathcal{A}_{1}, \ldots, \mathcal{A}_{m}\right\}$ of subcontinua of $\mu^{-1}(t)$ such that $P \in \mathcal{A}_{1}$ and $Q \in \mathcal{A}_{m}$. By Lemma 5.4, mesh $\left(\bigcup \mathcal{A}_{j}\right)<\varepsilon$ for all $j \in\{1, \ldots, m\}$. Since $P \in \mathcal{A}_{1} \cap \mathcal{C}(X)$, by [19, (1.49)], we have that $\bigcup \mathcal{A}_{1}$ is a subcontinuum of $X$. Hence, by Lemma 5.5 , we obtain that $\left(\cup \mathcal{A}_{1}\right) \cup\left(\cup \mathcal{A}_{2}\right)$ is also a subcontinuum of $X$. Continuing with this process we have that $\bigcup_{j=1}^{m}\left(\bigcup \mathcal{A}_{j}\right)$ is a subcontinuum of $X$. Now, by Lemma 5.5 , there exist a chain $\left\{B_{1}, \ldots, B_{m}\right\}$ of subcontinua of $X$ such that $P \subset B_{1}, Q \subset B_{m}$, where $B_{1}=\bigcup \mathcal{A}_{1}, B_{j}$ is a component of $\bigcup \mathcal{A}_{j}$ for each $j \in\{2, \ldots, m-1\}$ and $B_{m}=\bigcup \mathcal{A}_{m}\left(\bigcup \mathcal{A}_{m}\right.$ is connected by $[19,(1.49)]$, since $\left.Q \in \mathcal{A}_{m} \cap \mathcal{C}(X)\right)$. Since for every $j \in\{1, \ldots, m\}$, mesh $\left(\bigcup \mathcal{A}_{j}\right)<\varepsilon$, then $\operatorname{diam}\left(B_{j}\right)<\varepsilon$ for each $j \in\{1, \ldots, m\}$. Therefore, $X$ is a continuum chainable continuum. 
A topological property $P$ is a sequential powerful strong size-reversible property provided that whenever $X$ is a continuum for which there exist a positive integer $n$, a strong size map $\mu: \mathcal{C}_{n}(X) \rightarrow[0,1]$ and a sequence $\left\{t_{m}\right\}_{m=1}^{\infty}$ of elements of $(0,1)$ converging to 0 such that $\mu^{-1}\left(t_{m}\right)$ has property $P$ for all $m$. Then $X$ has property $P$.

The proof of the following theorem is similar to the one given for $[19$, (14.47)].

TheOREM 5.7. Let $X$ be a continuum, let $n$ be a positive integer and let $\mu: \mathcal{C}_{n}(X) \rightarrow[0,1]$ be a strong size map. If there exists a sequence $\left\{t_{m}\right\}_{m=1}^{\infty}$ of numbers in $[0,1)$ converging to 0 and $\mu^{-1}\left(t_{m}\right)$ is locally connected for each positive integer $m$, then $X$ is locally connected. Hence, being a locally connected continuum is a sequential powerful strong size-reversible property.

Proof. To show that $X$ is locally connected, by $[20,8.4]$, we need to prove that for each $\varepsilon>0, X$ can be written as the union of finitely many subcontinua each of diameter less than $\varepsilon$.

Let $\varepsilon>0$. By Lemma 5.3, there exists $t \in[0,1)$ such that $\operatorname{mesh}(A)<\varepsilon$ for all $A \in \mu^{-1}(t)$. Since $\left\{t_{m}\right\}_{m=1}^{\infty}$ converges to 0 , there exists $m$ such that $t_{m}<t$. Hence, $\operatorname{mesh}(A)<\varepsilon$ for all $A \in \mu^{-1}\left(t_{m}\right)$. Since $\mu^{-1}\left(t_{m}\right)$ is a locally connected continuum, by $[20,8.4]$, there exist finitely many subcontinua $\Gamma_{1}, \ldots, \Gamma_{k}$ of $\mu^{-1}\left(t_{m}\right)$ such that $\mu^{-1}\left(t_{m}\right)=\bigcup_{j=1}^{k} \Gamma_{j}$ and $\operatorname{diam}\left(\Gamma_{j}\right)<\frac{\varepsilon}{3 n}$ for each $j \in$ $\{1, \ldots, k\}$. For every $j \in\{1, \ldots, k\}$, let $G_{j}=\bigcup \Gamma_{j}$. Then, by [16, 6.1.2], $G_{j} \in \mathcal{C}_{n}(X)$ for all $j \in\{1, \ldots, k\}$. By Lemma $5.4, \operatorname{mesh}\left(G_{j}\right)<\varepsilon$ for every $j \in\{1, \ldots, k\}$. Since $\mu^{-1}\left(t_{m}\right)=\bigcup_{j=1}^{k} \Gamma_{j}$ and $\bigcup \mu^{-1}\left(t_{m}\right)=X$, we have that $X=\bigcup_{j=1}^{k} G_{j}$. Hence, $X$ can be written as the union of at most $n \cdot k$ subcontinua each of which has diameter less than $\varepsilon$. Therefore, $X$ is locally connected.

The proof of the following theorem is similar to the one given for $[14,50.4]$

THEOREM 5.8. The property of Kelley is a sequential powerful strong size-reversible property.

Proof. Let $X$ be a continuum, let $n$ be a positive integer and let $\mu: \mathcal{C}_{n}(X) \rightarrow[0,1]$ be a strong size map. Suppose there exists a sequence $\left\{t_{m}\right\}_{m=1}^{\infty}$ of elements of $[0,1]$ converging to 0 such that $\mu^{-1}\left(t_{m}\right)$ has the property of Kelley for each $m$.

Suppose that $X$ does not have the property of Kelley. Then there exist an element $p$ of $X$ and $\varepsilon>0$ such that for each positive integer $k$, there exist a point $q_{k}$ of $X$ and a subcontinuum $P_{k}$ of $X$ such that $d\left(p, q_{k}\right)<\frac{1}{k}, p \in P_{k}$ and for each subcontinuum $Q$ of $X$ containing $q_{k}$, we have that $\mathcal{H}\left(P_{k}, Q\right) \geq \varepsilon$. By Lemma 5.3, there exists $s \in[0,1]$ such that $\operatorname{mesh}(A)<\frac{\varepsilon}{3}$ for all $A \in \mu^{-1}(s)$. Let $N$ be a positive integer such that $t_{N}<s$. Note that $\operatorname{mesh}(A)<\frac{\varepsilon}{3}$ for every $A \in \mu^{-1}\left(t_{N}\right)$. 
For each positive integer $k$, let $Q_{k} \in \mu^{-1}\left(t_{N}\right) \cap \mathcal{C}(X)$ be such that $q_{k} \in Q_{k}$. Since $\mathcal{C}(X)$ is compact $([16,1.8 .5])$, without loss of generality, we assume that the sequences $\left\{P_{k}\right\}_{k=1}^{\infty}$ and $\left\{Q_{k}\right\}_{k=1}^{\infty}$ converge to $P$ and $Q$, respectively. Observe that $P$ and $Q$ are subcontinua of $X, Q \in \mu^{-1}\left(t_{N}\right)$ and $p \in P \cap Q$. Hence, $P \cup Q$ is a subcontinuum of $X$. Since $\operatorname{diam}(Q)=\operatorname{mesh}(Q)<\frac{\varepsilon}{3}$, we have that $\mathcal{H}(P, P \cup Q)<\frac{\varepsilon}{3}$.

Since $\mu^{-1}\left(t_{N}\right)$ has the property of Kelley, and $Q \in \mu^{-1}\left(t_{N}\right)$, there exists $\delta>0$ such that if $A \in \mu^{-1}\left(t_{N}\right), \mathcal{H}(Q, A)<\delta$ and $\mathcal{Q}$ is a subcontinuum of $\mu^{-1}\left(t_{N}\right)$ containing $Q$, there exists a subcontinuum $\mathcal{A}$ of $\mu^{-1}\left(t_{N}\right)$ such that $A \in \mathcal{A}$ and $\mathcal{H}^{2}(\mathcal{Q}, \mathcal{A})<\frac{\varepsilon}{3}$, where $\mathcal{H}^{2}$ is the Hausdorff metric on $\mathcal{C}\left(\mu^{-1}\left(t_{N}\right)\right)$.

Let $\mathcal{Q}=\mathcal{C}(P \cup Q) \cap \mu^{-1}\left(t_{N}\right)$. Thus, $\mathcal{Q}$ is a subcontinuum of $\mu^{-1}\left(t_{N}\right)$ containing $Q$. Let $k_{0}$ be a positive integer such that $\mathcal{H}\left(P_{k_{0}}, P\right)<\frac{\varepsilon}{3}$ and $\mathcal{H}\left(Q_{k_{0}}, Q\right)<\delta$. Then there exists a subcontinuum $\mathcal{Q}_{k_{0}}$ of $\mu^{-1}\left(t_{N}\right)$ such that $Q_{k_{0}} \in \mathcal{Q}_{k_{0}}$ and $\mathcal{H}^{2}\left(\mathcal{Q}, \mathcal{Q}_{k_{0}}\right)<\frac{\varepsilon}{3}$.

Let $Q^{\prime}=\bigcup \mathcal{Q}_{k_{0}}$. Then, by [19, (1.49)], $Q^{\prime}$ is a subcontinuum of $X$. Note that $q_{k_{0}} \in Q^{\prime}$. Since $P \cup Q=\bigcup \mathcal{Q}$ and $\mathcal{H}^{2}\left(\mathcal{Q}, \mathcal{Q}_{k_{0}}\right)<\frac{\varepsilon}{3}$, by [19, (1.48)], we obtain that $\mathcal{H}\left(P \cup Q, Q^{\prime}\right)<\frac{\varepsilon}{3}$. Hence, $\mathcal{H}\left(P_{k_{0}}, Q^{\prime}\right) \leq \mathcal{H}\left(P_{k_{0}}, P\right)+\mathcal{H}(P, P \cup$ $Q)+\mathcal{H}\left(P \cup Q, Q^{\prime}\right)<\varepsilon$, a contradiction to the election of $q_{k_{0}}$. Therefore, $X$ has the property of Kelley.

COROLlary 5.9. The property of Kelley is a powerful strong sizereversible property.

\section{Admissible Strong Size MAPS}

In [9], Professors J. T. Goodykoontz and S. B. Nadler, Jr. define admissible Whitney maps and prove that under the appropriate hypothesis, the Whitney levels of admissible Whitney maps are Hilbert cubes $([9,(4.1)])$. In this section we follow [9] and consider admissible strong size maps, and prove that under the appropriate hypothesis, the strong size levels of admissible strong size maps are also Hilbert cubes (Theorem 6.22). We also present other properties of admissible strong size maps.

Let $X$ be a continuum and let $n$ be a positive integer. A strong size map $\mu: \mathcal{C}_{n}(X) \rightarrow[0,1]$ is an admissible strong size map for $\mathcal{C}_{n}(X)$ provided that there exists a homotopy $H: \mathcal{C}_{n}(X) \times[0,1] \rightarrow \mathcal{C}_{n}(X)$ satisfying the following conditions:

(1) For all $A \in \mathcal{C}_{n}(X), H((A, 1))=A$ and $H((A, 0)) \in \mathcal{F}_{1}(X)$.

(2) If $\mu((H(A, t)))>0$ for some $A \in \mathcal{C}_{n}(X)$ and some $t \in(0,1]$, then $\mu(H((A, s)))<\mu(H((A, t)))$ for each $s \in[0, t)$.

A homotopy $H: \mathcal{C}_{n}(X) \times[0,1] \rightarrow \mathcal{C}_{n}(X)$, satisfying these conditions is called a $\mu$-admissible deformation for $\mathcal{C}_{n}(X)$.

Proposition $6.1([9,(2.2)])$. Let $X$ and $Y$ be homeomorphic continua and let $n$ be a positive integer. If there is an admissible strong size map $\mu$ for $\mathcal{C}_{n}(X)$, then there is an admissible strong size map for $\mathcal{C}_{n}(Y)$. 
Proof. Let $\mu_{X}$ be an admissible strong size map for $\mathcal{C}_{n}(X)$, and let $H_{X}$ be a $\mu_{X}$-admissible deformation for $\mathcal{C}_{n}(X)$. Let $h: Y \rightarrow X$ be a homeomorphism. Let $\mu_{Y}: \mathcal{C}_{n}(Y) \rightarrow[0,1]$ be given by $\mu_{Y}(B)=\mu_{X}\left(\mathcal{C}_{n}(h)(B)\right)$ for each $B \in \mathcal{C}_{n}(Y)$. Since $\mathcal{C}_{n}(h)$ is a homeomorphism ([5, Theorem 46]), $\mu_{Y}$ is a strong size map for $\mathcal{C}_{n}(Y)$. Now, let $H_{Y}: \mathcal{C}_{n}(Y) \times[0,1] \rightarrow \mathcal{C}_{n}(Y)$ be given by

$$
H_{Y}((B, t))=\mathcal{C}_{n}(h)^{-1}\left(H_{X}\left(\left(\mathcal{C}_{n}(h)(B), t\right)\right)\right)
$$

for all $(B, t) \in \mathcal{C}_{n}(Y) \times[0,1]$.

Next, we show that $H_{Y}$ is a $\mu_{Y}$-admissible deformation. To this end, let $B \in \mathcal{C}_{n}(Y)$. Then

$$
H_{Y}((B, 1))=\mathcal{C}_{n}(h)^{-1}\left(H_{X}\left(\left(\mathcal{C}_{n}(h)(B), 1\right)\right)\right)=\mathcal{C}_{n}(h)^{-1}\left(\left(\mathcal{C}_{n}(h)(B)\right)\right)=B .
$$

Since $H_{X}\left(\left(\mathcal{C}_{n}(h)(B), 0\right)\right) \in \mathcal{F}_{1}(X)$ and $\mathcal{C}_{n}(h)$ is an induced homeomorphism, we have that $\mathcal{C}_{n}(h)^{-1}\left(H_{X}\left(\left(\mathcal{C}_{n}(h)(B), 0\right)\right)\right) \in \mathcal{F}_{1}(Y)$. Thus, $H_{Y}((B, 0)) \in$ $\mathcal{F}_{1}(Y)$.

Now, let $B \in \mathcal{C}_{n}(Y)$ and let $t \in(0,1]$ be such that $\mu_{Y}\left(H_{Y}((B, t))\right)>0$ and let $s<t$. Then

$$
\begin{aligned}
\mu_{Y}( & \left.H_{Y}((B, s))\right)=\mu_{X}\left(\mathcal{C}_{n}(h)\left(H_{Y}((B, s))\right)\right) \\
& =\mu_{X}\left(\mathcal{C}_{n}(h)\left(\mathcal{C}_{n}(h)^{-1}\left(H_{X}\left(\left(\mathcal{C}_{n}(h)(B), s\right)\right)\right)\right)\right) \\
& =\mu_{X}\left(H_{X}\left(\left(\mathcal{C}_{n}(h)(B), s\right)\right)\right)<\mu_{X}\left(H_{X}\left(\left(\mathcal{C}_{n}(h)(B), t\right)\right)\right) \\
& =\mu_{X}\left(\mathcal{C}_{n}(h)\left(\mathcal{C}_{n}(h)^{-1}\left(H_{X}\left(\left(\mathcal{C}_{n}(h)(B), t\right)\right)\right)\right)\right) \\
& =\mu_{X}\left(\mathcal{C}_{n}(h)\left(H_{Y}((B, t))\right)\right)=\mu_{Y}\left(H_{Y}((B, t))\right) .
\end{aligned}
$$

Therefore, $H_{Y}$ is a $\mu_{Y}$-admissible deformation.

Theorem $6.2([9,(2.3)])$. Let $X$ be a continuum and let $n$ be a positive integer. If there is an admissible strong size map $\mu$ for $\mathcal{C}_{n}(X)$, then $\mathcal{F}_{n}(X)$ and $X$ are arcwise connected.

Proof. Let $H$ be a $\mu$-admissible deformation for $\mathcal{C}_{n}(X)$. Since $\mathcal{C}_{n}(X)$ is arcwise connected $([16,1.8 .12]), H\left(\mathcal{C}_{n}(X) \times\{0\}\right)$ is an arcwise connected subset of $\mathcal{F}_{n}(X)$ by condition (1) of the definition of an admissible strong size map. Let $A \in \mathcal{F}_{n}(X)$ and let $\mathcal{A}=\{H((A, t)) \mid t \in[0,1]\}$. Note that, by condition (1) of the definition of an admissible strong size map, $H((A, 1))=A$. Hence, $\mu(H((A, 1)))=0$. Thus, by condition (2) of the definition of an admissible strong size map, $\mathcal{A} \subset \mathcal{F}_{n}(X)$. As a consequence of this, since $\mathcal{A}$ is arcwise connected and $H((A, 0)), A \in \mathcal{A}$, there exists an arc in $\mathcal{F}_{n}(X)$ joining $H((A, 0)) \in \mathcal{A} \cap H\left(\mathcal{C}_{n}(X) \times\{0\}\right)$ and $H((A, 1))=A$. Therefore, $\mathcal{F}_{n}(X)$ is arcwise connected. Now, by [6, Proposition 2.7], $X$ is arcwise connected.

TheOREM $6.3([9,(2.4)])$. Let $X$ be a continuum and let $n$ be a positive integer, and let $\mu$ be an admissible strong size map for $\mathcal{C}_{n}(X)$. If $\mathcal{C}_{n}(X)$ is contractible, then $\mathcal{F}_{n}(X)$ is contractible. 
Proof. Suppose that $\mathcal{C}_{n}(X)$ is contractible. Then there exists a map $G^{\prime}: \mathcal{C}_{n}(X) \times[0,1] \rightarrow \mathcal{C}_{n}(X)$ such that $G^{\prime}((A, 0))=A$ and $G^{\prime}((A, 1))=X$. Let $G: \mathcal{C}_{n}(X) \times[0,1] \rightarrow \mathcal{C}_{n}(X)$ be the segment homotopy associated with $G^{\prime}$ defined by

$$
G((A, t))=\bigcup\left\{G^{\prime}((A, s)) \mid s \in[0, t)\right\} .
$$

Then $G$ is continuous $([19,(16.3)])$. Note that for each $A \in \mathcal{C}_{n}(X)$, $G((A, 0))=A, G((A, 1))=X$ and $G((A, \cdot)):[0,1] \rightarrow \mathcal{C}_{n}(X)$ is an order arc. Observe that, by [6, Proposition 2.6], $G$ is well defined.

Let $H$ be a $\mu$-admissible deformation for $\mathcal{C}_{n}(X)$. Let $L: \mathcal{F}_{n}(X) \times[0,1] \rightarrow$ $\mathcal{F}_{n}(X)$ be given by:

$$
L((A, t))= \begin{cases}H((A, 1-2 t)), & \text { if } t \in\left[0, \frac{1}{2}\right] \\ H((G(A, 2 t-1)), 0)), & \text { if } t \in\left[\frac{1}{2}, 1\right] .\end{cases}
$$

To see that the image of $L$ is contained in $\mathcal{F}_{n}(X)$, let $A \in \mathcal{F}_{n}(X)$ and let $s \in\left[0, \frac{1}{2}\right)$. Then $\mu(H((A, 1-2 s))=0$, otherwise $0<\mu(H((A, 1-2 s))<$ $\mu\left(H\left(\left(A, 1-2 \frac{1}{2}\right)\right)=\mu(H((A, 1-1))=\mu(H((A, 0)))=0\right.$, a contradiction. Therefore, $H((A, 1-2 s)) \in \mathcal{F}_{n}(X)$. If $A \in \mathcal{F}_{n}(X)$ and $s \in\left[\frac{1}{2}, 1\right]$, by the properties of $H, H((A, 1-2 s)) \in \mathcal{F}_{n}(X)$. Since for each $A \in \mathcal{F}_{n}(A), L((A, 0))=A$ and $L((A, 1))=H((X, 0))$, we have that $\mathcal{F}_{n}(X)$ is contractible.

Since the $n$-fold hyperspaces of continua with the property of Kelley are contractible $([16,6.1 .16])$, as a consequence of Theorem 6.3 , we obtain:

Corollary 6.4. Let $X$ be a continuum with the property of Kelley and let $n$ be a positive integer. If there exists an admissible strong size map $\mu$ for $\mathcal{C}_{n}(X)$, then $\mathcal{F}_{n}(X)$ are contractible.

Since locally connected continua have the property of Kelley [19, (16.11)], by Corollary 6.4 , we have:

Corollary 6.5. Let $X$ be a locally connected continuum and let $n$ be a positive integer. If there exists an admissible strong size map $\mu$ for $\mathcal{C}_{n}(X)$, then $X$ and $\mathcal{F}_{n}(X)$ are contractible.

COROllary $6.6([9,(2.6)])$. Let $X$ be a continuum that is an absolute neighborhood retract and let $n$ be a positive integer. If there exists an admissible strong size map $\mu$ for $\mathcal{C}_{n}(X)$, then $\mathcal{F}_{n}(X)$ is an absolute retract.

Proof. Since $X$ is an absolute neighborhood retract, by $[15$, Theorem $5.1], \mathcal{F}_{n}(X)$ is an absolute neighborhood retract. It follows from $[2,(1.1)$, p. 100] that $X$ is locally connected. Thus, since there exists an admissible strong size map for $\mathcal{C}_{n}(X)$, by Corollary $6.5, \mathcal{F}_{n}(X)$ is contractible. Hence, by $[2,9.1$, p. 96$], \mathcal{F}_{n}(X)$ is an absolute retract. 
A metric $\rho$ for a continuum $X$ is said to be convex provided that given to points $x_{1}$ and $x_{2}$ of $X$, there exists a third point $x_{3}$ in $X$ such that $\rho\left(x_{1}, x_{3}\right)=$ $\rho\left(x_{2}, x_{3}\right)=\frac{1}{2} \rho\left(x_{1}, x_{2}\right)$.

A proof of the following result may be found in [1] and [18].

THEOREM 6.7. Every locally connected continuum admits a convex metric.

REMARK 6.8. If $X$ is a locally connected continuum with a convex metric $\rho$, let $K_{\rho}:[0, \infty) \times 2^{X} \rightarrow 2^{X}$ be given by

$$
K_{\rho}((t, A))=\{x \in X \mid \rho(x, a) \leq t \text { for some } a \in A\} .
$$

Note that, by $[19,(0.65 .3(\mathrm{f}))]$, is continuous. Also observe that, by [16, 6.7.12], we have that $K_{\rho}((t, A)) \in \mathcal{C}_{n}(X)$ for every $A \in \mathcal{C}_{n}(X)$ and each $t \in[0, \infty)$. In fact, if $A \in 2^{X}$, then $K_{\rho}((\cdot, A)):[0, \infty) \rightarrow 2^{X}$ is an order arc. Hence, $\left.K_{\rho}\right|_{[0, \infty) \times \mathcal{C}_{n}(X)}:[0, \infty) \times \mathcal{C}_{n}(X) \rightarrow \mathcal{C}_{n}(X)$ is well defined. $K_{\rho}$ is continuous by $[19,(0.65 .3)(\mathrm{f})]$.

THEOREM 6.9. Let $X$ be locally connected continuum with a convex metric $\rho$, let $n$ be a positive integer, let $\mu: \mathcal{C}_{n}(X) \rightarrow[0,1]$ be a strong size map, and let $t \in[0,1]$. Then $\mu^{-1}(t)$ is a strong deformation retract of $\mu^{-1}[0, t]$.

Proof. Let $A \in \mu^{-1}[0, t]$. Then, since $K_{\rho}((\cdot, A)):[0, \infty) \rightarrow \mathcal{C}_{n}(X)$ is an order arc (Remark 6.8), there exists a unique element $r_{A}$ in $[0, \infty)$ such that $K_{\rho}\left(\left(r_{A}, A\right)\right) \in \mu^{-1}(t)$. Let $\mathfrak{M}: \mu^{-1}[0, t] \rightarrow \mathbb{R}$ be given by $\mathfrak{M}(A)=$ $r_{A}$. To see that $\mathfrak{M}$ is continuous, let $\left\{A_{k}\right\}_{k=1}^{\infty}$ be a sequence of elements of $\mu^{-1}([0, t])$ that converges to a point $A$ of $\mu^{-1}([0, t])$. We need to show that the sequence $\left\{r_{A_{k}}\right\}_{k=1}^{\infty}$ converges to $r_{A}$. Suppose that $\left\{r_{A_{k}}\right\}_{k=1}^{\infty}$ converges to $\ell$. Then, since $K_{\rho}$ is continuous $([19,(0.65 .3)(\mathrm{f})])$, we have that the sequence $\left\{K_{\rho}\left(\left(r_{A_{k}}, A_{k}\right)\right)\right\}_{k=1}^{\infty}$ converges to $K_{\rho}((\ell, A))$. Since for each positive integer $k, K_{\rho}\left(\left(r_{A_{k}}, A_{k}\right)\right)$ belongs to $\mu^{-1}(t)$, we obtain that $K_{\rho}((\ell, A))$ also belongs to $\mu^{-1}(t)$. Thus, $\ell=r_{A}$. Therefore, $\mathfrak{M}$ is continuous. Note that if $A \in \mu^{-1}(t)$, then $\mathfrak{M}(A)=0$.

Let $H: \mu^{-1}[0, t] \times[0,1] \rightarrow \mu^{-1}[0, t]$ be given by $H((A, s))=K_{\rho}((s$. $\mathfrak{M}(A), A))$. Then $H$ is continuous. Let $A \in \mu^{-1}[0, t]$. Then $H((A, 0))=$ $K_{\rho}((0 \cdot \mathfrak{M}(A), A))=K_{\rho}((0, A))=A, H((A, 1))=K_{\rho}((1 \cdot \mathfrak{M}(A), A))=$ $K_{\rho}((\mathfrak{M}(A), A)) \in \mu^{-1}(t)$. Furthermore, if $A \in \mu^{-1}(t)$ and $s \in[0,1]$, then $H((A, s))=K_{\rho}((s \cdot \mathfrak{M}(A), A))=K_{\rho}((s \cdot 0, A))=K_{\rho}((0, A))=A$. Therefore, $\mu^{-1}(t)$ is a strong deformation retract of $\mu^{-1}[0, t]$.

Corollary 6.10. Let $X$ be a locally connected continuum with a convex metric $\rho$, let $n$ be a positive integer, and let $\mu: \mathcal{C}_{n}(X) \rightarrow[0,1]$ be a strong size map. Then $\mu^{-1}([t, 1])$ is an absolute retract for every $t \in[0,1]$.

Proof. Let $t \in[0,1]$. If $t=1$, then $\mu^{-1}(1)=\{X\}$. Hence, $\mu^{-1}(1)$ is an absolute retract. If $t=0$, then $\mu^{-1}([0,1])=\mathcal{C}_{n}(X)$. Since $\mathcal{C}_{n}(X)$ is 
an absolute retract $\left(\left[23\right.\right.$, Théorème $\left.\left.\mathrm{II}_{m}\right]\right)$, we are done. Suppose $t \in(0,1)$ by Theorem $6.9, \mu^{-1}(t)$ is a strong deformation retract of $\mu^{-1}[0, t]$. Since $\mu^{-1}([0, t]) \cap \mu^{-1}([t, 1])=\mu^{-1}(t)$, we have that $\mu^{-1}([t, 1])$ is a strong deformation retract of $\mathcal{C}_{n}(X)$. Thus, since $\mathcal{C}_{n}(X)$ is an absolute retract, we have that $\mu^{-1}([t, 1])$ is an absolute retract $([2,(2.2)$, p. 86$])$.

TheOREM $6.11([9,(2.7)])$. Let $X$ be a continuum and let $n$ be a positive integer. If $\mu$ is an admissible strong size map for $\mathcal{C}_{n}(X)$, then for each $t_{0} \in$ $(0,1), \mu^{-1}\left(t_{0}\right)$ is a retract of $\mu^{-1}\left(\left[t_{0}, 1\right]\right)$.

Proof. Let $H: \mathcal{C}_{n}(X) \times[0,1] \rightarrow \mathcal{C}_{n}(X)$ be a $\mu$-admissible deformation for $\mathcal{C}_{n}(X)$. Let $t_{0} \in(0,1)$, and let $A \in \mu^{-1}\left(\left[t_{0}, 1\right]\right)$. Since $H((A, 1))=A$, $\mu(H((A, 1))) \geq t_{0}$. Also, since $H((A, 0)) \in \mathcal{F}_{1}(X), \mu(H((A, 0)))=0$. Hence, there exists an element $s_{A}$ of $[0,1]$ such that $\mu\left(H\left(\left(A, s_{A}\right)\right)\right)=t_{0}$. Note that, by the properties of $H, s_{A}$ is unique. Let $\mathfrak{N}: \mu^{-1}\left(\left[t_{0}, 1\right]\right) \rightarrow[0, \infty)$ be given by $\mathfrak{N}(A)=s_{A}$. To see that $\mathfrak{N}$ is continuous, let $\left\{A_{k}\right\}_{k=1}^{\infty}$ be a sequence of elements of $\mu^{-1}\left(\left[t_{0}, 1\right]\right)$ converging to a point $A$ of $\mu^{-1}\left(\left[t_{0}, 1\right]\right)$. We need to prove that the sequence $\left\{s_{A_{k}}\right\}_{k=1}$ converges to $s_{A}$. Suppose that $\left\{s_{A_{k}}\right\}_{k=1}$ converges to $\ell$. Since $H$ is continuous, the sequence $\left\{H\left(\left(A, s_{A_{k}}\right)\right)\right\}_{k=1}^{\infty}$ converges to $H((A, \ell))$. Since for each positive integer $k, H\left(\left(A, s_{A_{k}}\right)\right)$ belongs to $\mu^{-1}\left(t_{0}\right)$, we have that $H((A, \ell))$ belongs to $\mu^{-1}\left(t_{0}\right)$ too. Thus, $\ell=s_{A}$. Therefore, $\mathfrak{N}$ is continuous. Observe that if $A \in \mu^{-1}\left(t_{0}\right)$, then $\mathfrak{N}(A)=0$.

Let $r: \mu^{-1}\left(\left[t_{0}, 1\right]\right) \rightarrow \mu^{-1}\left(t_{0}\right)$ be given by $r(A)=H((A, 1-\mathfrak{N}(A)))$. Then $r$ is continuous. If $A \in \mu^{-1}\left(t_{0}\right)$, then $\mathfrak{N}(A)=0$. Thus, $r(A)=H((A, 1-$ $\mathfrak{N}(A))=H((A, 1))=A$. Therefore, $r$ is a retraction from $\mu^{-1}\left(\left[t_{0}, 1\right]\right)$ onto $\mu^{-1}\left(t_{0}\right)$.

TheOREM $6.12([9,(2.8)])$. Let $X$ be a continuum, let $n$ be a positive integer, and let $\mu$ be an admissible strong size map for $\mathcal{C}_{n}(X)$. If $\mathcal{C}_{n}(X)$ is contractible, then $\mu^{-1}\left(t_{0}\right)$ is contractible for each $t_{0} \in[0,1]$.

Proof. Let $t_{0} \in[0,1]$. If $t=0$, then $\mu^{-1}\left(t_{0}\right)=\mathcal{F}_{n}(X)$ and, by Theorem $6.3, \mu^{-1}\left(t_{0}\right)$ is contractible. Since $\mu^{-1}(1)=\{X\}$, we have that $\mu^{-1}(1)$ is contractible too. Assume that $t_{0} \in(0,1)$.

Since $\mathcal{C}_{n}(X)$ is contractible, there exists a map $G^{\prime}: \mathcal{C}_{n}(X) \times[0,1] \rightarrow \mathcal{C}_{n}(X)$ such that $G^{\prime}((A, 0))=A$ and $G^{\prime}((A, 1))=X$. Let $G: \mathcal{C}_{n}(X) \times[0,1] \rightarrow \mathcal{C}_{n}(X)$ be the segment homotopy associated with $G^{\prime}$ defined by

$$
G((A, t))=\bigcup\left\{G^{\prime}((A, s)) \mid s \in[0, t)\right\} .
$$

Then $G$ is continuous ([19, (16.3)]). Note that for each $A \in \mathcal{C}_{n}(X)$, $G((A, 0))=A, G((A, 1))=X$ and $G((A, \cdot)):[0,1] \rightarrow \mathcal{C}_{n}(X)$ is an order arc. Observe that, by [6, Proposition 2.6], $G$ is well defined. Note that $G\left(\mu^{-1}\left(\left[t_{0}, 1\right]\right) \times[0,1]\right)=\mu^{-1}\left(\left[t_{0}, 1\right]\right)$. Hence, $\mu^{-1}\left(\left[t_{0}, 1\right]\right)$ is contractible. Since, by Theorem $6.11, \mu^{-1}\left(t_{0}\right)$ is a retract of $\mu^{-1}\left(\left[t_{0}, 1\right]\right)$, we have that $\mu^{-1}\left(t_{0}\right)$ is contractible $([2,(13.2)$, p. 26]). 
Theorem $6.13([9,(2.9)])$. Let $X$ be a locally connected continuum with a convex metric $\rho$, let $n$ be a positive integer, and let $\mu: \mathcal{C}_{n}(X) \rightarrow[0,1]$ be a strong size map. Then $\mu^{-1}\left(t_{0}\right)$ is an absolute retract for each $t_{0} \in(0,1)$.

Proof. Let $t_{0} \in(0,1)$. By Corollary $6.10, \mu^{-1}\left(\left[t_{0}, 1\right]\right)$ is an absolute retract. Also, by Theorem $6.11, \mu^{-1}\left(t_{0}\right)$ is a retract of $\mu^{-1}\left(\left[t_{0}, 1\right]\right)$. Hence, by $[2,(2.2)$, p. 86$], \mu^{-1}\left(t_{0}\right)$ is an absolute retract.

TheOREM $6.14([9,(2.13)])$. Let $X$ be a continuum with metric $d$ and let $n$ be a positive integer. Suppose that there exists a homotopy $\varphi: X \times[0,1] \rightarrow X$ satisfying the following conditions: $x \in X$;

(1) $\varphi((x, 1))=x$ and, for some fixed point $p \in X, \varphi((x, 0))=p$ for every

(2) If $d\left(\varphi\left(\left(x_{1}, t\right)\right), \varphi\left(\left(x_{2}, t\right)\right)\right)>0$ for $x_{1}, x_{2} \in X$ and $t \in[0,1]$, then $d\left(\varphi\left(\left(x_{1}, s\right)\right), \varphi\left(\left(x_{2}, s\right)\right)\right)<d\left(\varphi\left(\left(x_{1}, t\right)\right), \varphi\left(\left(x_{2}, t\right)\right)\right)$ for all $s \in[0, t)$.

Then there exists an admissible strong size map $\omega$ for every $\mathcal{C}_{n}(X)$.

Proof. Without loss of generality, we assume that $\operatorname{diam}(X)=1$. Define $\omega: \mathcal{C}_{n}(X) \rightarrow[0,1]$, as $\omega(A)=\operatorname{diam}(A) \cdot \mu(A)$, where $\mu$ is the strong size map defined in Example 4.2. By Lemma $4.3, \omega$ is a strong size map. Define, $H: \mathcal{C}_{n}(X) \times[0,1] \rightarrow \mathcal{C}_{n}(X)$, by $H((A, t))=\varphi(A \times\{t\})$. Since $\varphi$ is uniformly continuous map, $H$ is continuous. We show that $H$ is an $\omega$-admissible deformation for $\mathcal{C}_{n}(X)$. To this end, let $A \in \mathcal{C}_{n}(X)$. Then $H((A, 1))=\varphi(A \times\{1\})=A$ and $H((A, 0))=\varphi(A \times\{0\})=\{p\} \in \mathcal{F}_{1}(X)$.

Let $A \in \mathcal{C}_{n}(X)$ and let $t \in[0,1]$ be such that $\omega(H((A, t)))>0$. Let $s \in[0, t)$. If $s=0$, then $\omega(H((A, s)))=0<\omega(H((A, t)))$. Assume that $s>0$. Observe that for every $m \geq n$, we have that $\mu_{m}(H((A, t))) \geq \mu_{m}(H((A, s)))$, since, if $H((A, t)) \subset \mathcal{V}_{\varepsilon}\left(\left\{p_{1}, \ldots, p_{m}\right\}\right)$, then, by definition of $\varphi$, we obtain that:

$$
H((A, s)) \subset \mathcal{V}_{\varepsilon}\left(\left\{\varphi\left(\left(p_{1}, s\right)\right), \ldots, \varphi\left(\left(p_{m}, s\right)\right)\right\}\right) .
$$

Thus, $\mu(H((A, t))) \geq \mu(H((A, s)))$. Now, by the definition of $H$, we have that $\operatorname{diam}(H((A, t)))>\operatorname{diam}(H((A, s)))$. Hence, we have that $\omega(H((A, t)))>$ $\omega(H((A, s)))$.

A subcontinuum $S$ of a Banach space $E$ is starshaped if there exists an element $p$ in $S$ such that for every $x \in S$, the convex arc in $E$ from $p$ to $x$ is contained in $S$.

Corollary $6.15([9,(2.14)])$. Let $X$ be a starshaped subcontinuum of a Banach space $E$ and let $n$ be a positive integer. Then there exists an admissible strong size map $\mu$ for $\mathcal{C}_{n}(X)$.

The proof of the following corollary is the same as the one given in $[9$, (2.15)] since the authors embed $X$ as starshaped subcontinuum of a Banach space. 
Corollary $6.16([9,(2.15)])$. Let $X$ be the topological cone over a nonempty compact metric space $Y$ and let $n$ be a positive integer. Then there exists an admissible strong size map $\mu$ for $\mathcal{C}_{n}(X)$.

The proof of the following theorem is the same as the one given in $[9$, (2.16)], the authors construct a map satisfying the requirements of Theorem 6.14 .

Theorem $6.17([9,(2.16)])$. If $X$ is a dendrite and $n$ is a positive integer, then there exists an admissible strong size map $\mu$ for $\mathcal{C}_{n}(X)$.

Proposition $6.18([9,(3.1)])$. Let $X$ be a continuum and let $n$ be a positive integer. Let $\mu$ be a strong size map for $\mathcal{C}_{n}(X)$, let $\mathcal{E} \subset \mathcal{C}_{n}(X)$ and let $\sigma: \mathcal{E} \rightarrow \mathcal{C}\left(\mathcal{C}_{n}(X)\right)$ be a map such that for every $B \in \mathcal{E}, \sigma(B)$ is an order arc in $\mathcal{C}_{n}(X)$. Let $t_{0} \in[0,1]$, and assume that for each $B \in \mathcal{E}, \mu^{-1}\left(t_{0}\right) \cap \sigma(B) \neq \emptyset$. Then for each $B \in \mathcal{E}, \sigma(B) \cap \mu^{-1}\left(t_{0}\right)$ consists of exactly one point of $\mu^{-1}\left(t_{0}\right)$, denoted by $\sigma_{t_{0}}(B)$, and the function $\sigma_{t_{0}}: \mathcal{E} \rightarrow \mu^{-1}\left(t_{0}\right)$ is continuous.

Proof. Let $B \in \mathcal{E}$. Since $\sigma(B)$ is an order arc, if $\mu^{-1}\left(t_{0}\right) \cap \sigma(B) \neq \emptyset$, then $\mu^{-1}\left(t_{0}\right) \cap \sigma(B)$ consists of exactly one point of $\mu^{-1}\left(t_{0}\right)$. To show that $\sigma_{t_{0}}$ is continuous, let $\left\{B_{m}\right\}_{m=1}^{\infty}$ be a sequence of elements of $\mathcal{E}$ converging to an element $B$ of $\mathcal{E}$. Since $\mu^{-1}\left(t_{0}\right)$ is compact, without loss of generality, we assume that $\left\{\sigma_{t_{0}}\left(B_{m}\right)\right\}_{m=1}^{\infty}$ converges to a point $A$ of $\mu^{-1}\left(t_{0}\right)$. Since $\sigma$ is continuous, $A \in \sigma(B)$. Thus, $A \in \mu^{-1}\left(t_{0}\right) \cap \sigma(B)$. Hence, $A=\sigma_{t_{0}}(B)$. Therefore, $\sigma_{t_{0}}$ is continuous.

Lemma 6.19 ([7, 5.4]). Let $X$ be a nondegenerate locally connected continuum, let $A$ be a closed subset of $X$ without free arcs in $X$ such that $A$ has nonempty interior in $X$, let $n$ be a positive integer and let $\varepsilon>0$. Then there exists a map $R: \mathcal{C}_{n}(X) \rightarrow \mathcal{C}_{n}(X) \backslash \mathcal{C}_{n}(A, X)$ such that $\rho_{0}\left(R, 1_{\mathcal{C}_{n}(X)}\right)<\varepsilon$, where $\rho_{0}$ is the "sup" metric, and $R(B) \backslash A=B \backslash A$ for every $B \in \mathcal{C}_{n}(X)$.

Proof. Since $X$ is a locally connected continuum, there exists a finite collection $\mathfrak{P}$ of locally connected subcontinua of $X$ such that the diameter of each element of $\mathfrak{P}$ is smaller that $\frac{1}{2} \varepsilon$, and each element of $\mathfrak{P}$ is the closure of an open connected subset of $X([20,8.9])$. Without loss of generality, we assume that there exists an element $P$ of $\mathfrak{P}$ such that:

$$
S t(P)=\bigcup\left\{P^{\prime} \in \mathfrak{P} \mid P^{\prime} \cap P \neq \emptyset\right\}
$$

is contained in $A$. Since $P$ is a locally connected continuum, there exists a tree $T$ contained in $P$ such that

$$
M=T \cup \bigcup\left\{P^{\prime} \in \mathfrak{P} \mid P^{\prime} \cap P \neq \emptyset \text { and } P^{\prime} \neq P\right\}
$$

is connected. Hence, $M$ is locally connected. This implies that $\mathcal{C}_{n}(M)$ is an absolute retract $\left(\left[23\right.\right.$, Théorème $\left.\left.\mathrm{II}_{m}\right]\right)$. Thus, there exists a map $r_{0}: S t(P) \rightarrow$ 
$\mathcal{C}_{n}(M)$ such that $r_{0}(x)=\{x\}$ for all $x \in M$. We extend $r_{0}$ to a map $r: X \rightarrow$ $\mathcal{C}_{n}(X)$ by setting $r(x)=\{x\}$ for every $x \in X \backslash S t(P)$.

Since $A$ does not contain free arcs, we have that $\operatorname{Int}(P)$ is not contained in $M$. Define $R: \mathcal{C}_{n}(X) \rightarrow \mathcal{C}_{n}(X) \backslash \mathcal{C}_{n}(A, X)$ by

$$
R(B)=\bigcup\{r(b) \mid b \in B\} .
$$

Note that, by construction, $R$ is well defined, $R(B) \backslash A=B \backslash A$ for all $B \in \mathcal{C}_{n}(X)$ and $\rho_{0}\left(R, 1_{\mathcal{C}_{n}(X)}\right)<\varepsilon$.

Lemma $6.20([9,(3.2)])$. Let $X$ be a locally connected continuum and let $A$ be closed subset of $X$ such that $A$ has nonempty interior. Assume that $A$ does not contain any free arc in $X$. If $\mu$ is an admissible strong size map for $\mathcal{C}_{n}(X)$, then for each $t_{0} \in(0,1)$, the set $\left\{B \in \mu^{-1}\left(t_{0}\right) \mid A \subset B\right\}$ is a Z-set in $\mu^{-1}\left(t_{0}\right)$.

Proof. Let $H: \mathcal{C}_{n}(X) \times[0,1] \rightarrow \mathcal{C}_{n}(X)$ be a $\mu$-admissible deformation. Let $t_{0}$ and $s$ be two elements of $(0,1)$. Define $R: \mathcal{C}_{n}(X) \rightarrow \mathcal{C}_{n}(X)$ as in Lemma 6.19; i.e. $R(B)=\bigcup\{r(b): b \in B\}$ for each $B \in \mathcal{C}_{n}(X)$. Hence, we have the following:

(1) If $B_{1}, B_{2} \in \mathcal{C}_{n}(X)$ and $B_{1} \subset B_{2}$, then $R\left(B_{1}\right) \subset R\left(B_{2}\right)$.

By Lemma 6.19 , we assume that $R$ is close enough to the identity map such that:

(2) $\mu(R(X))>t_{0}$.

Since $H$ is a $\mu$-admissible deformation for $\mathcal{C}_{n}(X)$ and, since $s<1$ and $t_{0}>0$, $\mu(H((B, s)))<t_{0}$ for each $B \in \mu^{-1}\left(t_{0}\right)$. Hence, since $\mu^{-1}\left(t_{0}\right)$ is compact and $\mu$ and $H$ are continuous functions:

$$
\inf \left\{t_{0}-\mu(H((B, s))) \mid B \in \mu^{-1}\left(t_{0}\right)\right\}>0 .
$$

Therefore, by Lemma 6.19 , we may assume that $R$ is near enough to the identity map such that:

(3) $\mu(R(H((B, s))))<t_{0}$ for every $B \in \mu^{-1}\left(t_{0}\right)$.

Since $X$ is locally connected, $X$ admits a convex metric $\rho$ ([1] or [18]), and consider the map $K_{\rho}:[0, \infty) \times \mathcal{C}_{n}(X) \rightarrow \mathcal{C}_{n}(X)$ given in Remark 6.8. For each $B \in \mu^{-1}\left(t_{0}\right)$ let

$$
\sigma(B)=\left\{R\left(K_{\rho}((t, H((B, s))))\right) \mid t \geq 0\right\} .
$$

Since $K_{\rho}$ and $R$ are continuous, we have that $\sigma(B)$ is a subcontinuum of $\mathcal{C}_{n}(X)$ for all $B \in \mu^{-1}\left(t_{0}\right)$. Also, by (1), we obtain that:

$$
R\left(K_{\rho}\left(\left(t_{1}, H((B, s))\right)\right)\right) \subset R\left(K_{\rho}\left(\left(t_{2}, H((B, s))\right)\right)\right) \text { if } 0 \leq t_{1} \leq t_{2} .
$$

Hence, by $[19,(1.4)]$ and $[6,2.6], \sigma(B)$ is an order arc in $\mathcal{C}_{n}(X)$ for each $B \in \mu^{-1}\left(t_{0}\right)$. Since $H, K_{\rho}$ and $R$ are continuous, it follows that $\sigma: \mathcal{C}_{n}(X) \rightarrow$ $\mathcal{C}\left(\mathcal{C}_{n}(X)\right)$ is continuous too. For each $B \in \mu^{-1}\left(t_{0}\right)$, by (3), we have:

$$
\mu\left(R\left(K_{\rho}((0, H((B, s))))\right)\right)<t_{0},
$$


and letting $\gamma=\operatorname{diam}(X)$, we obtain, by $(2)$ :

$$
\mu\left(R\left(K_{\rho}((\gamma, H((B, s))))\right)\right)>t_{0} .
$$

Hence, since for every $B \in \mu^{-1}\left(t_{0}\right), \sigma(B)$ is an order arc, we have that $\sigma(B) \cap$ $\mu^{-1}\left(t_{0}\right) \neq \emptyset$ for all $B \in \mu^{-1}\left(t_{0}\right)$. Let $\sigma_{t_{0}}: \mu^{-1}\left(t_{0}\right) \rightarrow \mu^{-1}\left(t_{0}\right)$ be given as in Proposition 6.18. Then $\sigma_{t_{0}}$ is continuous and, by Lemma 6.19, $A \not \subset R(B)$ for any $B \in \mathcal{C}_{n}(X)$. This implies that $\sigma_{t_{0}}$ maps $\mu^{-1}\left(t_{0}\right)$ into $\mu^{-1}\left(t_{0}\right) \backslash\{B \in$ $\left.\mu^{-1}\left(t_{0}\right) \mid A \subset B\right\}$. Also: for any given $\varepsilon>0$, it is not difficult to see that if $s$ is close enough to 1 and $R$ is near enough of $1_{\mathcal{C}_{n}(X)}$, then $\rho_{0}\left(\sigma_{t_{0}}, 1_{\mu^{-1}\left(t_{0}\right)}\right)<\varepsilon$. Therefore, $\left\{B \in \mu^{-1}\left(t_{0}\right) \mid A \subset B\right\}$ is a $Z$-set in $\mu^{-1}\left(t_{0}\right)$.

The following result is a special case of [21, Theorem 1].

THEOREM 6.21. Let $Y$ be an absolute retract. If $1_{Y}$ is a uniform limit of $Z$-maps, then $Y$ is a Hilbert cube.

THEOREM $6.22([9,(4.1)])$. Let $X$ be locally connected continuum that does not contain free arcs and let $n$ be a positive integer. If there exist an admissible strong size map $\mu$ for $\mathcal{C}_{n}(X)$, then $\mu^{-1}\left(t_{0}\right)$ is a Hilbert cube for every $t_{0} \in(0,1)$.

Proof. Let $H: \mathcal{C}_{n}(X) \times[0,1] \rightarrow \mathcal{C}_{n}(X)$ be a $\mu$-admissible deformation for $\mathcal{C}_{n}(X)$ and let $t_{0} \in(0,1)$. By Theorem 6.13 , we have that $\mu^{-1}\left(t_{0}\right)$ is an absolute retract. Hence, by Theorem 6.21 , it suffices to show that $1_{\mu^{-1}\left(t_{0}\right)}$ is a uniform limit of $Z$-maps. Since $X$ is a locally connected continuum, there exist a convex metric $\rho$ for $X$ (see [1] or [18]) and consider the map $K_{\rho}:[0, \infty) \times \mathcal{C}_{n}(X) \rightarrow \mathcal{C}_{n}(X)$ given in Remark 6.8. Let $s \in(0,1)$. Define $\sigma: \mu^{-1}\left(t_{0}\right) \rightarrow \mathcal{C}_{1}\left(\mathcal{C}_{n}(X)\right)$ by

$$
\sigma(B)=\left\{K_{\rho}((t,(H((B, s))))) \mid t \geq 0\right\}
$$

for each $B \in \mu^{-1}\left(t_{0}\right)$. Since $H$ and $K_{\rho}$ are continuous, with a similar argument to the one given in the proof of Lemma 6.20, we have that $\sigma$ is continuous. Note that for every $B \in \mu^{-1}\left(t_{0}\right), \sigma(B)$ is an order arc $([6,2.6])$. Thus, $\sigma(B) \cap \mu^{-1}\left(t_{0}\right) \neq \emptyset$. Hence, we may define $\sigma_{t_{0}}: \mu^{-1}\left(t_{0}\right) \rightarrow \mu^{-1}\left(t_{0}\right)$ as in Proposition 6.18. We show that $\sigma_{t_{0}}$ is a $Z$-map.

Since $H$ is a $\mu$-admissible deformation and since $s<1$ and $t_{0}>0$, $\mu(H((B, s)))<t_{0}$ for each $B \in \mu^{-1}\left(t_{0}\right)$. Thus, since $\mu^{-1}\left(t_{0}\right)$ is compact and $H$ and $\mu$ are continuous, we have that

$$
\sup \left\{\mu(H((B, s))) \mid B \in \mu^{-1}\left(t_{0}\right)\right\}<t_{0} .
$$

By the definition of $\sigma_{t_{0}}$, we have that for each $B \in \mu^{-1}\left(t_{0}\right)$, there exists $t_{B}$ such that $\sigma_{t_{0}}(B)=\mu\left(K_{\rho}\left(\left(t_{B}, H((B, s))\right)\right)\right)$. Since, $\mu\left(\sigma_{t_{0}}(B)\right)=t_{0}$ for each $B \in \mu^{-1}\left(t_{0}\right)$, it follows that there exists $\gamma>0$ such that $t_{B} \geq \gamma$ for every $B \in \mu^{-1}\left(t_{0}\right)$. Let $\left\{p_{1}, \ldots, p_{m}\right\}$ be a finite subset of $X$ such that 
$\mathcal{H}\left(X,\left\{p_{1}, \ldots, p_{m}\right\}\right)<\frac{\gamma}{2}$. For each $j \in\{1, \ldots, m\}$, let

$$
\mathcal{P}_{j}=\left\{E \in \mu^{-1}\left(t_{0}\right) \mid K_{\rho}\left(\left(\frac{\gamma}{2},\left\{p_{j}\right\}\right)\right) \subset E\right\} .
$$

Now, we show that $\sigma_{t_{0}}\left(\mu^{-1}\left(t_{0}\right)\right) \subset \bigcup_{j=1}^{m} \mathcal{P}_{j}$. Let $B_{0} \in \mu^{-1}\left(t_{0}\right)$ and let $x_{0} \in$ $H\left(\left(B_{0}, s\right)\right)$. Then there exists $p_{j} \in\left\{p_{1}, \ldots, p_{m}\right\}$ such that $\rho\left(x_{0}, p_{j}\right)<\frac{\gamma}{2}$. For every $y \in K_{\rho}\left(\left(\frac{\gamma}{2},\left\{p_{j}\right\}\right)\right)$, it follows that

$$
\rho\left(y, x_{0}\right) \leq \rho\left(y, p_{j}\right)+\rho\left(p_{j}, x_{0}\right) \leq \gamma \leq t_{B_{0}} .
$$

Hence, $K_{\rho}\left(\left(\frac{\gamma}{2},\left\{p_{j}\right\}\right)\right) \subset K_{\rho}\left(\left(t_{B_{0}}, H\left(\left(B_{0}, s\right)\right)\right)\right)=\sigma_{t_{0}}\left(B_{0}\right)$. Thus, $\sigma_{t_{0}}\left(\mu^{-1}\left(t_{0}\right)\right)$ $\subset \bigcup_{j=1}^{m} \mathcal{P}_{j}$. Note that, by Lemma 6.20 , each $\mathcal{P}_{j}$ is a $Z$-set in $\mu^{-1}\left(t_{0}\right)$. Hence, $\sigma_{t_{0}}\left(\mu^{-1}\left(t_{0}\right)\right)$ is contained in a finite union of $Z$-sets of $\mu^{-1}\left(t_{0}\right)$. Thus, by [4, Theorem 3.1], $\sigma_{t_{0}}\left(\mu^{-1}\left(t_{0}\right)\right)$ is a $Z$-set of $\mu^{-1}\left(t_{0}\right)$. Therefore, $\sigma_{t_{0}}$ is a $Z$ map. It is not difficult to see that if $s$ had been sufficiently close to 1 , then $\sigma_{t_{0}}$ would have been as near to $1_{\sigma_{t_{0}}}$ as desire. Thus, we have proved that $1_{\sigma_{t_{0}}}$ is a uniform limit of $Z$-maps. Therefore, by Theorem $6.21, \mu^{-1}\left(t_{0}\right)$ is a Hilbert cube.

\section{About the existence of Strong size Preserving Maps for $n \geq 2$}

In [8] the notion of Whiney preserving functions is introduced. We show that the corresponding notion for strong size maps and for an integer $n$ greater than or equal to two is trivial; i.e., that type of map must be a homeomorphism (Theorem 7.4).

Let $X$ and $Y$ be continua and let $n$ be an integer greater than or equal to 2. A map $f: X \rightarrow Y$ is an $n$-strong size preserving map if there exist two strong size maps

$$
\mu: \mathcal{C}_{n}(X) \rightarrow[0,1] \text { and } \omega: \mathcal{C}_{n}(Y) \rightarrow[0,1]
$$

such that for each $t \in[0,1]$, there exists $s \in[0,1]$ such that

$$
\mathcal{C}_{n}(f)\left(\mu^{-1}(t)\right)=\omega^{-1}(s)
$$

This means that a map $f$ is an $n$-strong preserving map, if there exist two strong size maps for which the induced map $\mathcal{C}_{n}(f)$ maps strong size levels of $\mathcal{C}_{n}(X)$ onto strong size levels of $\mathcal{C}_{n}(Y)$. In this case, we say that $f$ is $(\mu, \omega)$-n-strong size preserving.

LEMMA 7.1. Let $X$ and $Y$ be continua and let $n$ be an integer greater than or equal to 2 . If $f: X \rightarrow Y$ is an $(\mu, \omega)$-n-strong size preserving map, then $f$ is surjective and $\mathcal{C}_{n}(f)\left(\mu^{-1}(0)\right)=\omega^{-1}(0)$.

Proof. Let $f: X \rightarrow Y$ be an $n$-strong size preserving map. Let $\left\{x_{1}, \ldots, x_{m}\right\} \in \mu^{-1}(0)$. Clearly, $\mathcal{C}_{n}(f)\left(\left\{x_{1}, \ldots, x_{m}\right\}\right)=\left\{f\left(x_{1}\right), \ldots, f\left(x_{m}\right)\right\} \in$ $\omega^{-1}(0)$. Hence, $\mathcal{C}_{n}(f)\left(\mu^{-1}(0)\right) \subset \omega^{-1}(0)$. By definition of $n$-strong size preserving map, $\mathcal{C}_{n}(f)\left(\mu^{-1}(0)\right)=\omega^{-1}(t)$ for some $t \in[0,1]$. Since $\mathcal{C}_{n}(f)\left(\mu^{-1}(0)\right) \cap \omega^{-1}(0) \neq \emptyset$, we obtain that $\mathcal{C}_{n}(f)\left(\mu^{-1}(0)\right)=\omega^{-1}(0)$. 
Let $y \in Y$. Then $\{y\} \in \omega^{-1}(0)$. Hence, by the previous paragraph, there exists $A \in \mu^{-1}(0)$ such that $\mathcal{C}_{n}(f)(A)=\{y\}$. Clearly, if $a \in A$, then $f(a)=y$. Therefore, $f$ is surjective.

Lemma 7.2. Let $X$ and $Y$ be continua such that $Y$ is nondegenerate and let $n$ be an integer greater than or equal to 2 . If $f: X \rightarrow Y$ is $a(\mu, \omega)$-n-strong size preserving map, then $f$ is light.

Proof. Suppose the map $f$ is not light. Let

$$
\mathcal{A}=\left\{\mu(A) \mid A \in \mathcal{C}(X) \text { and } \mathcal{C}_{n}(f)(A) \in \mathcal{F}_{1}(X)\right\} .
$$

Since $f$ is not light, $\mathcal{A}$ is nonempty, also since $Y$ is nondegenerate, we have that $\max \mathcal{A}>0$. Let $a=\max \mathcal{A}$, let $A \in \mu^{-1}(a)$, and let $p \in X \backslash A$. Since $\mu$ is a strong size map, $\mu(A \cup\{p\})>\mu(A)$; however $\mathcal{C}_{n}(f)(A \cup\{p\}) \in \mathcal{F}_{n}(Y)$. This implies that $\omega\left(\mathcal{C}_{n}(f)(A \cup\{p\})\right)=0$. Let $B$ a subcontinuum of $X$ such that $A \subsetneq B$ and $\mu(A \cup\{p\})>\mu(B)$. Then, since $A \in \mu^{-1}(a)$, we have that $\mathcal{C}_{n}(f)(B) \notin \mathcal{F}_{n}(X)$. Thus, $\omega\left(\mathcal{C}_{n}(f)(B)\right)>\omega\left(\mathcal{C}_{n}(f)(A \cup\{p\})\right.$, a contradiction. Therefore, $f$ is light.

Lemma 7.3. Let $X$ and $Y$ be two continua such that $Y$ is nondegenerate and let $n$ be an integer greater than or equal to 2 . Let $f: X \rightarrow Y$ be $a(\mu, \omega)$ $n$-strong size preserving map. If $\mathcal{C}_{n}(f)\left(\mu^{-1}(t)\right)=\omega^{-1}(s)$ and $t^{\prime} \in(0, t)$, then $\mathcal{C}_{n}(f)\left(\mu^{-1}\left(t^{\prime}\right)\right)=\omega^{-1}\left(s^{\prime}\right)$, where $s^{\prime} \leq s$.

Proof. Let $A^{\prime} \in \mu^{-1}\left(t^{\prime}\right)$ and let $A \in \mu^{-1}(t)$ be such that $A^{\prime} \subset A$. Then $\mathcal{C}_{n}(f)\left(A^{\prime}\right) \subset \mathcal{C}_{n}(f)(A)$. Hence, $\omega\left(\mathcal{C}_{n}(f)\left(A^{\prime}\right)\right) \leq \omega\left(\mathcal{C}_{n}(f)(A)\right)$. Therefore, $s^{\prime} \leq s$. Since $f$ is a $(\mu, \omega)$-n-strong size preserving map, we have that $\mathcal{C}_{n}(f)\left(\mu^{-1}\left(t^{\prime}\right)\right)=\omega^{-1}\left(s^{\prime}\right)$.

THEOREM 7.4. Let $X$ and $Y$ be two continua such that $Y$ is nondegenerate and let $n$ be an integer greater than or equal to 2 . If $f: X \rightarrow Y$ is $a(\mu, \omega)$ $n$-strong size preserving map, then $f$ is an homeomorphism.

Proof. Suppose that $f$ is not a homeomorphism. Since $f$ is $(\mu, \omega)$ $n$-strong size preserving map, by Lemma 7.1, $f$ surjective. Thus, $f$ is not one-to-one. Let $p$ and $q$ be two different points of $X$ such that $f(p)=f(q)$. Let $\alpha:[0,1] \rightarrow \mathcal{C}_{n}(X)$ be an order arc such that $\alpha(0)=\{p\}$ and $\alpha(1)=X$. Let $r_{q}=\min \{t \in[0,1] \mid q \in \alpha(t)\}$. Since $r_{q}>0$ and $f$ is light (Lemma 7.2), $\omega\left(\mathcal{C}_{n}(f)\left(\alpha\left(r_{q}\right)\right)\right)>0$. Let $s_{q}=\omega\left(\mathcal{C}_{n}(f)\left(\alpha\left(r_{q}\right)\right)\right)$. By the continuity of $\omega$ and $\mathcal{C}_{n}(f)$, and since $\mathcal{C}_{n}(f)(\{p, q\}) \in \mathcal{F}_{n}(Y)$, we have that there exists $r \in\left(0, r_{q}\right)$ such that

$$
0<\omega\left(\mathcal{C}_{n}(f)(\alpha(r) \cup\{q\})\right)<s_{q} .
$$

Since $\mu$ is a strong size map and $q \notin \alpha(r)$ and $\alpha(r) \notin \mathcal{F}_{n}(X), \mu(\alpha(r) \cup\{q\})>$ $\mu(\alpha(r))>0$. Let $A$ be the element of $\mu^{-1}(\mu(\alpha(r) \cup\{q\})) \cap \alpha([0,1])$ and assume that $\mu(A)=r_{\mu}$. 
By the continuity of $\alpha, \mathcal{C}_{n}(f)$ and $\mu$, there exists $r^{\prime} \in\left(r, r_{\mu}\right)$ such that

$$
\mu(\alpha(r) \cup\{q\})>\mu\left(\alpha\left(r^{\prime}\right)\right)>\mu(\alpha(r)) .
$$

Since $\alpha$ is an order arc, $\mathcal{C}_{n}(f)$ is an induced map and $\omega$ is a strong size map, we have that:

$$
\omega \circ \mathcal{C}_{n}(f) \circ \alpha:[0,1] \rightarrow[0,1]
$$

is an increasing map. Since $f$ is light (Lemma 7.2) and $\omega$ is a strong size map, $\left.\omega\left(\mathcal{C}_{n}(f)(\alpha(t))\right)\right)=0$ if and only if $t=0$. Thus, there exists $s^{\prime} \in\left(0, r^{\prime}\right)$ such that for all $s>s^{\prime}$, we obtain that

$$
\omega\left(\mathcal{C}_{n}(f)\left(\alpha\left(s^{\prime}\right)\right)\right)<\omega\left(\mathcal{C}_{n}(f)(\alpha(s))\right) .
$$

This implies that $\omega\left(\mathcal{C}_{n}(f)(\alpha(r) \cup\{q\})\right)=\omega\left(\mathcal{C}_{n}(f)(\alpha(r))\right)<\omega\left(\mathcal{C}_{n}(f)\left(\alpha\left(r^{\prime}\right)\right)\right)$, a contradiction to Lemma 7.3 , since $\mu(\alpha(r) \cup\{q\})>\mu\left(\alpha\left(r^{\prime}\right)\right)$.

\section{REFERENCES}

[1] R. H. Bing, Partitioning a set, Bull. Amer. Math. Soc. 55 (1949), 1101-1110.

[2] K. Borsuk, Theory of retracts, Monogr. Mat. 44, Państwowe Wydawnictwo Naukowe, Warsaw, 1967.

[3] K. Borsuk and S. Ulam, On symmetric products of topological spaces, Bull. Amer. Math. Soc. 37 (1931), 875-882.

[4] T. A. Chapman, Lectures on Hilbert cube manifolds, American Mathematical Society, Providence, 1976.

[5] J. J. Charatonik, A. Illanes and S. Macías, Induced mappings on the hyperspaces $\mathcal{C}_{n}(X)$ of a continuum X, Houston J. Math. 28 (2002), 781-805.

[6] J. J. Charatonik and S. Macías, Mappings of some hyperspaces, JP J. Geom. Topol. 4 (2004), 53-80.

[7] D. W. Curtis and R. M. Schori, Hyperspaces which characterize simple homotopy type, General Topology and Appl. 6 (1976), 153-165.

[8] B. Espinoza Reyes, Whitney preserving functions, Topology Appl. 126 (2002), 351358.

[9] J. T. Goodykoontz and S. B. Nadler, Jr., Whitney levels in hyperspaces of certain Peano continua, Trans. Amer. Math. Soc. 274 (1982), 671-694.

[10] H. Hosokawa, Induced mappings on hyperspaces, Tsukuba J. Math. 21 (1997), 239-250.

[11] H. Hosokawa, Strong size levels of $C_{n}(X)$, Houston J. Math. 37 (2011), 955-965.

[12] A. Illanes, Monotone and open Whitney maps, Proc. Amer. Math. Soc. 98 (1986), $516-518$.

[13] A. Illanes, S. Macías and S. B. Nadler, Jr., Symmetric products and $\mathcal{Q}$-manifolds, in Geometry and topology in dynamics, American Mathematical Society, Providence, 1999, 137-141.

[14] A. Illanes and S. B. Nadler, Jr. Hyperspaces. Fundamentals and recent advances, Marcel Dekker, New York, 1999.

[15] J. W. Jaworowski, Symmetric products of ANR's, Math. Ann. 192 (1971), 173-176.

[16] S. Macías, Topics on continua, Chapman \& Hall/CRC, Boca Raton, 2005.

[17] S. Macías and C. Piceno, Strong size properties, Glas. Mat. Ser. III 48(68) (2013), 103-114.

[18] E. E. Moise, Grille decomposition and convexification theorems for compact metric locally connected continua, Bull. Amer. Math. Soc. 55 (1949), 1111-1121. 
[19] S. B. Nadler, Jr., Hyperspaces of sets, Marcel Dekker, New York, Basel, 1978. Reprinted in: Aportaciones Matemáticas de la Sociedad Matemática Mexicana, Serie Textos \# 33, 2006.

[20] S. B. Nadler, Jr., Continuum theory: an introduction, Marcel Dekker, New York, Basel, Hong Kong, 1992.

[21] H. Toruńczyk, On CE-images of the Hilbert cube and characterization of $\mathcal{Q}$-manifolds, Fund. Math. 106 (1980), 31-40.

[22] H. Whitney, On regular families of curves I, Proc. Nat. Acad. Sci. 18 (1932), 275-278.

[23] M. Wojdisławski, Rétractes absolus et hyperspaces des continus, Fund. Math. 32 (1939), 184-192.

\section{S. Macías}

Instituto de Matemáticas

Universidad Nacional Autónoma de México

Circuito Exterior, Ciudad Universitaria, México D. F., C. P. 04510

México

E-mail: sergiom@matem.unam.mx

C. Piceno

Instituto de Matemáticas

Universidad Nacional Autónoma de México

Circuito Exterior, Ciudad Universitaria México D. F., C. P. 04510

México.

E-mail: cesarpicman@hotmail.com

Received: 24.1.2014.

Revised: 7.8.2014. 